\title{
Clinical Images An Abscess of Urachal Remnant
}

\author{
Yuta Hirose, MD, Kiyoshi Shikino, MD, PhD, and Masatomi lkusaka, MD, PhD \\ Department of General Medicine, Chiba University Hospital, Chiba-city, Chiba, Japan.
}

KEY WORDS: urachal remnant; urachus.

J Gen Intern Med 32(3):360

DOI: $10.1007 / \mathrm{s} 11606-016-3804-1$

(c) Society of General Internal Medicine 2016

A

43-year-old man presented with one week of abdominal

pain and umbilical discharge (Fig. 1). On exam, there was a painful mass in the infraumbilical region and a positive Carnett's sign (increased pain with tensing of the abdominal muscles suggestive of abdominal wall pathology). Urine test results were negative. Abdominal enhanced computed tomography showed a rim-enhancing mass and a linear lesion between the mass and bladder dome (Fig. 2). Culture of the umbilical discharge was positive for Peptoniphilus species. Urachal remnant abscess was diagnosed. After abscess drainage and intravenous antibiotics administration, the symptoms alleviated.

The urachus, a vestigial structure connecting the bladder dome to umbilicus, occludes and normally becomes a fibrous cord after birth. ${ }^{1}$ If occlusion is insufficient, an urachal remnant forms and may eventually become infected. Tenderness between the umbilicus and lower abdomen in conjunction with pus discharge from the umbilicus are indicative of a urachal remnant abscess. ${ }^{2}$ Urine test results are negative in

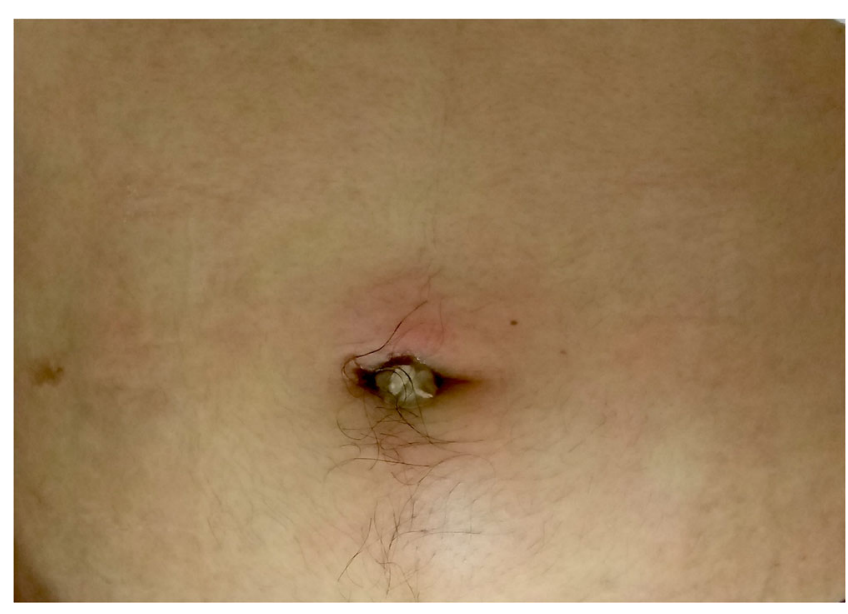

Figure 1. Pus discharge from the umbilicus and erythema around the umbilicus.

Received April 21, 2016

Revised June 3, 2016

Accepted June 27, 2016

Published online July 8, 2016

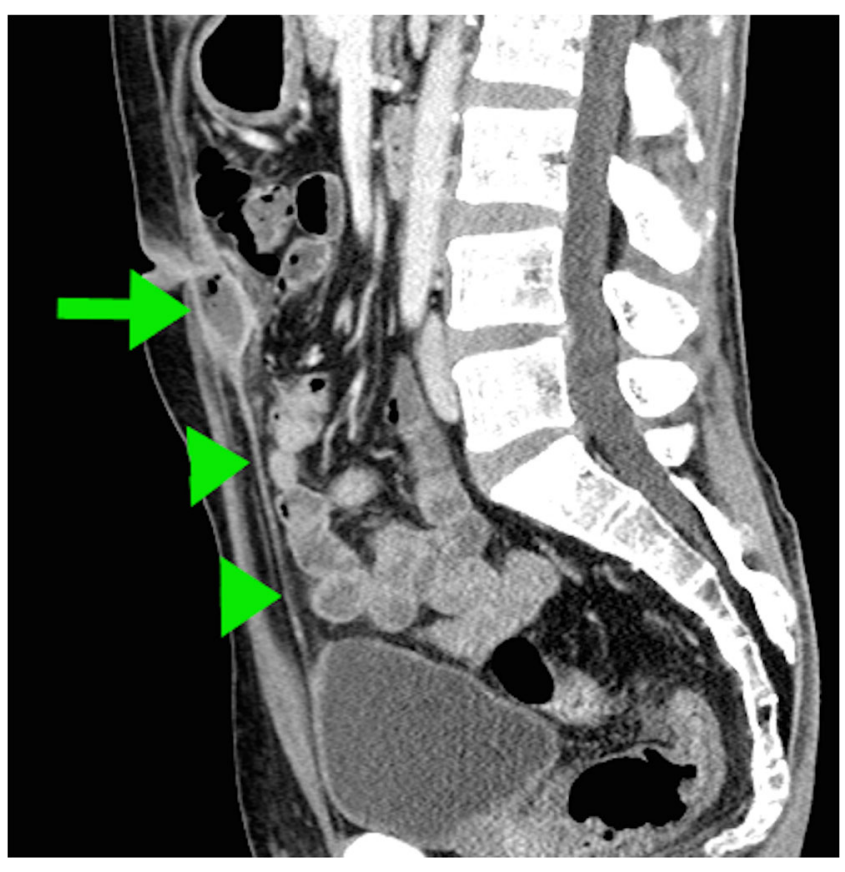

Figure 2. There is a mass containing air and covered by an enhanced wall (arrow). Linear lesion between the mass and the dome of the bladder (arrowheads).

more than $80 \%$ of cases. ${ }^{1}$ The differential diagnoses include "Umbilicus cellulitis" and a "Sister Mary Joseph's nodule. ${ }^{3}$ " After resolution of the infection, there are recommendations that the urachal remnant should be excised to prevent recurrent infection and avoid the risk of neoplastic transformation. ${ }^{1}$

Acknowledgements: We are grateful to Dr. Akinori Takei, Department of Urology, Chiba University Hospital, for managing the patient.

Corresponding Author: Yuta Hirose, MD; Department of General MedicineChiba University Hospital, 1-8-1, Inohana, Chuo-ku, Chibacity, Chiba, Japan (e-mail: yuta1076@yahoo.co.jp).

\section{Compliance with Ethical Standards:}

Conflict of Interest: The authors declare that they do not have a conflict of interest.

\section{REFERENCES}

1. Tazi F. Abscess of urachal remnants presenting with acute abdmen: a case series. J Med Case Rep. 2012;6:226-232.

2. EI Ammari JE. Urachal sinus presenting with abscess formation. ISRN Urol. 2011;2011:820924.

3. Miller T, Ashworth J, Richards S. Sister Mary Joseph nodule. BMJ. 2015;351:h5224. 